\title{
Prostaglandins and Radical Oxygen Species Are Involved in Microvascular Effects of Hyperoxia
}

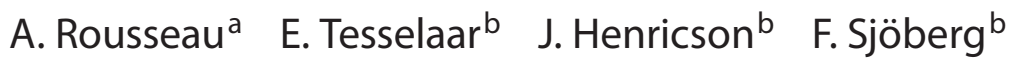 \\ Departments of a Medicine and Health Sciences, and ${ }^{\mathrm{b}} \mathrm{Clinical}$ and Experimental Medicine, Faculty of Health \\ Sciences, Linköping University, Linköping, Sweden
}

\section{Key Words}

Acetylcholine - Perfusion • Hyperoxia • lontophoresis •

Prostaglandins $\cdot$ Radical oxygen species $\cdot$ Sodium

nitroprusside

\begin{abstract}
Hyperoxia causes vasoconstriction in most tissues, by mechanisms that are not fully understood. We investigated microvascular effects of breathing $100 \%$ oxygen in healthy volunteers, using iontophoresis to deliver acetylcholine (ACh) and sodium nitroprusside (SNP). Aspirin and vitamin C were used to test for involvement of prostaglandins and radical oxygen species. Forearm skin perfusion was measured using laser Doppler perfusion imaging. Results were analysed using dose-response modelling. The response to $A C h$ was reduced by $30 \%$ during oxygen breathing compared to air breathing [0.98 (0.81-1.15) PU vs. 1.45 (1.30-1.60) PU, p < 0.001]. ED 50 values were unchanged [2.25 (1.84-2.75) vs. 2.21 (1.79-2.74), not significant]. Aspirin pre-treatment abolished the difference in response between oxygen breathing and air breathing [maximum: $1.03(0.90-1.16)$ vs. 0.89 (0.77-1.01), not significant; $E_{50}: 1.83$ (1.46-2.30) vs. 1.95 (1.65-2.30), not significant]. ACh-mediated vasodilatation during $100 \%$ oxygen breathing was partially restored after pre-treatment with vitamin C. Breathing $100 \%$ oxygen did not change the microvascular response to SNP [1.45 (1.28-1.62) vs. 1.40 (1.26-1.53), not significant]. These results favour the hypothesis that hy-
\end{abstract}

peroxic vasoconstriction is mediated by inhibition of prostaglandin synthesis. Radical oxygen species may be involved as vitamin C, independently of aspirin, partially restored ACh-mediated vasodilatation during hyperoxia.

Copyright $\odot 2010$ S. Karger AG, Basel

\section{Introduction}

Hyperoxia has cardiovascular effects in humans, causing a reduction in cardiac output and heart rate [1-4] as well as an increase in peripheral resistance and vascular tone $[5,6]$. As a result, perfusion is reduced in most tissues [7-9]. It is probable that hyperoxia acts firstly at the microvascular level by inducing peripheral vasoconstriction $[5,7]$. The reduction in cardiac output is a secondary effect and works to keep the blood pressure constant.

The mechanisms underlying the effect of oxygen in the microvasculature still have to be elucidated. Several theories have been presented, including the action of red blood cells as an oxygen sensor [10]. Red blood cells release increased amounts of adenosine 5'-triphosphate during hypoxia, which result in a vasodilator response. Oxygen may also induce changes in the rheology of red blood cells, which in itself modulates tissue perfusion [11]. In previous findings in rat cremaster arterioles and human umbilical arteries, alterations in endotheliumderived dilator prostanoids have been found to contrib-

\section{KARGER}

Fax +41613061234 E-Mail karger@karger.ch www.karger.com
(C) 2010 S. Karger AG, Basel

$1018-1172 / 10 / 0475-0441 \$ 26.00 / 0$

Accessible online at:

www.karger.com/jvr
Dr. Andréas Rousseau

Department of Medicine and Health Sciences

University Hospital of Linköping

SE-581 85 Linköping (Sweden)

Tel. +46 132220 00, Fax +46 132228 36, E-Mail andreas.rousseau@lio.se 
Table 1. Details of the subjects participating in the study

\begin{tabular}{llllll}
\hline Protocol & 1 & 2 & 3 & 4 & 5 \\
\hline Number $^{1}$ & $10(6)$ & $9(9)$ & $11(5)$ & $9(8)$ & $9(7)$ \\
Mean age, years $^{2}$ & $28(22-33)$ & $29(23-41)$ & $26(22-29)$ & $28(23-32)$ & $27(23-33)$ \\
$\begin{array}{l}\text { Mean BMI } \\
\text { Mean skin temperature }\end{array}$ & $22(2.0)$ & $23(2.1)$ & $23(3.3)$ & $22(1.5)$ & $22(2.6)$ \\
$\quad$ Air breathing & $31(1.7)$ & $32(0.7)$ & $31(1.1)$ & $30(0.9)$ & $32(1.0)$ \\
$\quad 100 \%$ oxygen breathing & $31(1.6)$ & $32(0.7)$ & $31(1.2)$ & $30(0.9)$ & $32(0.9)$ \\
\hline
\end{tabular}

$\mathrm{BMI}=$ Body mass index. ${ }^{1}$ Figures in parentheses are men. ${ }^{2}$ Figures in parentheses are ranges. ${ }^{3}$ Figures in parentheses are SD.

ute to hyperoxic vasoconstriction [12-14]. Also, the vasoconstricting effect of hyperoxia has been attributed to oxygen free radicals reacting with and inactivating the vasodilator agent nitric oxide (NO) $[2,4,15,16]$.

The primary aim of this study was to test the hypothesis that a brief period of substantial normobaric hyperoxia (100\% oxygen breathing during $10 \mathrm{~min}$ ) affects the vasoactive response in the microcirculation of the human forearm skin. The secondary aim was to investigate if any effect of normobaric hyperoxia is dependent on endothelial mechanisms and whether prostaglandins or oxygen free radicals are involved.

To answer these questions, we used a non-invasive technique. A laser Doppler perfusion imager (LDPI) [17] was used to measure perfusion in the skin of healthy subjects and iontophoresis [18] was used as a way of delivering drugs through intact skin. To be able to identify possible involvement of endothelium, acetylcholine (ACh, an endothelium-dependent vasodilator) and sodium nitroprusside (SNP, a direct NO donor) were used. Acetylsalicylic acid (aspirin) and vitamin $\mathrm{C}$ were used to test whether any effect of hyperoxia on microvascular responses in the forearm skin was dependent on prostaglandins or oxygen free radicals. Finally, sodium chloride was delivered to test for any non-specific effects of iontophoresis on forearm skin perfusion.

\section{Subjects and Methods}

\section{Subjects}

Twenty-two healthy volunteers took part in the study (15 men). The study was divided into 5 protocols depending on the drugs that were used (ACh alone, ACh with aspirin, ACh with vitamin C, SNP and sodium chloride as a control solution). The volunteers could participate in more than one protocol. Subjects were inter- viewed and a physical examination was made. Apart from 3 of the women who were taking oral contraceptives, the volunteers were not taking any drugs. Subjects were not included if they had either neurological, cardiovascular, pulmonary, hepatic, renal, haematopoietic, gastrointestinal or metabolic dysfunction. The volunteers were asked to refrain from coffee, tea and nicotine products for $10 \mathrm{~h}$ before the experiments. They were all fully informed of the nature and purpose of the study. The study was approved by the local ethics committee. Demographic details of the subjects are presented in table 1.

\section{Laser Doppler Perfusion Imaging}

An LDPI (PIM 1.0; Lisca Development AB, Linköping, Sweden) was used to measure perfusion in the skin. The laser beam, with a wavelength of $670 \mathrm{~nm}$, penetrates the skin and a fraction of light is backscattered, containing a frequency-shifted part and a non-frequency-shifted part. The frequency-shifted part is the light backscattered by mobile red blood cells (the Doppler principle). The relative contribution of the two parts decides the perfusion value, which is defined as the number of blood cells multiplied by the mean cell velocity. At each measuring site this procedure is repeated (up to 4,096 measurements) to form a colour-coded image on a computer screen.

To keep a constant distance between the head of the LDPI and the skin, two adjustable laser beams were attached to the head of the scanner. We chose $15 \mathrm{~cm}$ as an optimal distance, as this gives a reliable signal [17]. Before starting, the distance between the head of the scanner and the skin was measured with a ruler. Then the two laser beams were set to cross each other at skin level. For each new measurement, the laser beam cross-hair was used to set the scanner head quickly at a constant distance.

Perfusion measurements were made with the LDPI in duplex mode, scanning the measuring site continuously. In duplex mode, the measurement value generated by the software (LDPIwin v2.3.13; Lisca Development $A B$ ) is the geometrical mean of the $4 \times 4$ measurement points.

\section{Iontophoresis}

After the flexor side of one of the forearms had been gently cleaned with an ethanol wipe, a customized iontophoresis electrode chamber (Lisca Development AB, ) was attached to the skin by double-sided adhesive Scotch tape. The area of measurement 
was selected to avoid visible veins and pigmented naevi. The inner diameter of the ring-shaped chamber was $15 \mathrm{~mm}$, and the distance between the silver-silver chloride electrode ring and the skin was $2 \mathrm{~mm}$. The electrode chamber was filled with the drug solution (about $430 \mu \mathrm{l}$ ) and covered by a thin glass slide. The head of the scanner was tilted somewhat to avoid direct reflection of the laser beam from the glass slide into the head. An indifferent electrode (Perimed AB, Stockholm, Sweden) was applied to the wrist. A battery-powered iontophoresis controller (PeriIont 382; Perimed AB) was connected to both electrodes to deliver a constant electrical current to the skin. The subjects were told to hold their arms still during the experiment. Skin temperature (digital thermometer; Claes Ohlson, Insjon, Sweden) was recorded before and after each measurement on the same forearm, about $5 \mathrm{~cm}$ proximal to the measuring site. When more than one measurement was performed in the same subject, different skin sites were used for each measurement.

\section{Drugs}

ACh chloride (Miochol ${ }^{\circledR}$-E $10 \mathrm{mg} / \mathrm{ml}$; Novartis Healthcare, Copenhagen, Denmark), SNP (Nitropress ${ }^{\circledR} 25 \mathrm{mg} / \mathrm{ml}$; Abbott, Chicago, Ill., USA), acetylsalicylic acid (Aspirin ${ }^{\circledR} 500 \mathrm{mg}$; Bayer $\mathrm{AB}$, Solna, Sweden), vitamin C (C-vimin ${ }^{\circledR}$ 1g; AstraZeneca, Södertälje, Sweden) and sodium chloride $(9 \mathrm{mg} / \mathrm{ml})$, were dispensed by the hospital pharmacy. ACh (1\%) and SNP (1\%) were dissolved in a sodium chloride solution such that a final ionic strength of $154 \mathrm{~mm}$ was obtained [19]. Drug concentrations were the same throughout the experiments. All drugs were prepared immediately before being used and SNP was kept in the dark.

\section{Air and Oxygen}

Medical air and $100 \%$ oxygen were given through a non-rebreathing system, with the subjects breathing through a facemask. The subjects did not know whether air or oxygen was being given.

The subjects started to breathe air from the facemask $10 \mathrm{~min}$ before the measurements started, to get used to the mask. Thereafter, air was administered during the period of iontophoresis, after which oxygen was given for the same amount of time. Oxygen was always given last to avoid washout effects of oxygen during the measurements made with air.

\section{Experimental Protocol}

The subjects rested lying down for $30 \mathrm{~min}$ in a temperaturecontrolled room at $23^{\circ} \mathrm{C}(0.6)$. A thin cotton blanket was placed over the trunk and lower extremities to prevent cooling. All measurements were made in a dark room, the only light coming from the screen connected to the LDPI. All measurements were made with the subjects lying down.

In previous studies we optimised our iontophoresis protocols to reduce the vasodilatating effect of the current alone and to maximise the response to the drugs, with most responses ending as plateaus $[19,20]$. For all measurement protocols in this study, we used a single pulse at a constant current. The duration of the pulse was $10 \mathrm{~min}$ and the current strength was $0.02 \mathrm{~mA}$.

The current study was divided into 5 protocols. In protocol 1 $(\mathrm{n}=10)$, ACh was delivered to the skin by anodal iontophoresis. In protocol $2(\mathrm{n}=9)$, SNP was delivered by cathodal iontophoresis. In protocol $3(\mathrm{n}=11)$, ACh was delivered after $2 \times 500 \mathrm{mg}$ of acetylsalicylic acid had been given $24 \mathrm{~h}$ before the measurements, followed by another $2 \times 500 \mathrm{mg}$ of acetylsalicylic acid $2 \mathrm{~h}$ before the measurements. In protocol $4(n=9)$, iontophoresis of ACh was preceded by the intake of vitamin C. Vitamin C $(2.5 \mathrm{~g})$ was given by mouth every morning for 3 consecutive days. The last dose was taken 1-2 $\mathrm{h}$ before the examination took place [21]. In protocol 5 $(n=9)$, sodium chloride was delivered by cathodal iontophoresis. The protocols were performed on separate days and at different skin sites.

\section{Dose-Response Model}

The perfusion response from each individual subject was plotted as a function of the logarithm of electrical charge. The electrical charge is indicative of the dose of drugs given during iontophoresis $[22,23]$.

The data were analysed by fitting the $\mathrm{E}_{\max }$ model, based on classical receptor occupancy theory [24], to the pooled perfusion data in every group of measurements. The model can be described by the following equation:

$$
E(t)=E_{\min }+\frac{\left(E_{\max }-E_{\min }\right) C(t)^{n}}{C(t)^{n}+E D_{50}^{n}}
$$

where $\mathrm{E}_{\min }$ is the baseline, $\mathrm{E}_{\max }$ is the maximum perfusion, $\mathrm{ED}_{50}$ is the iontophoretic charge eliciting half of the maximum response and $\mathrm{n}$ is the Hill slope, which defines the steepness of the response. $\mathrm{C}(\mathrm{t})$ is the electrical charge as a function of time, defined as the current strength multiplied by the pulse duration: $\mathrm{C}(\mathrm{t})=\mathrm{I} \times \mathrm{t}$.

In each protocol, the last $10 \mathrm{~s}$ before the start of the first iontophoresis period (with air breathing) was considered as baseline perfusion. This baseline value was subtracted from all further data points obtained during both iontophoresis periods (with both air breathing and oxygen breathing).

\section{Statistics}

The minimum perfusion $\left(\mathrm{E}_{\min }\right)$, the maximum response $\left(\mathrm{E}_{\max }-\mathrm{E}_{\min }\right)$, the $\mathrm{ED}_{50}$ and their respective confidence intervals ( $95 \%$ CI) were estimated from each response by fitting the model to the pooled data in each protocol. Since baseline perfusion was subtracted, $\mathrm{E}_{\min }$ was fixed to zero for the air breathing data. Data were analysed using GraphPad Prism version 5.00 for Windows (GraphPad Software, San Diego, Calif., USA). Data in the figures are presented as means \pm SEM. For clarity, a limited number of data points are shown in the figures.

The significance of the difference in these estimated values between air breathing and oxygen breathing was tested using an Ftest. Probabilities of less than 0.05 were accepted as significant.

\section{Results}

During all protocols where ACh and SNP were delivered, an increase in skin perfusion was found, which almost always resulted in plateaus in the responses. Fitting the model to the obtained perfusion data yielded well-defined dose-response curves in all protocols $\left(\mathrm{R}^{2}\right.$ values ranged from 0.55 to 0.95 ). An overview of the best-fit parameters and comparisons is presented in table 2 . 
Table 2. Overview of dose response parameters for all protocols (increase in perfusion, $\mathrm{ED}_{50}$ )

\begin{tabular}{|c|c|c|c|c|}
\hline & Protocol & Air & $100 \% \mathrm{O}_{2}$ & Air vs. $100 \% \mathrm{O}_{2}$ \\
\hline \multicolumn{5}{|c|}{ Maximum response [PU] } \\
\hline 1 & $\mathrm{ACh}$ & $1.45(1.30-1.60)$ & $0.98(0.81-1.15)$ & $\mathrm{p}<0.001$ \\
\hline 2 & SNP & $1.45(1.28-1.62)$ & $1.40(1.26-1.53)$ & NS \\
\hline 3 & $\mathrm{ACh}+\mathrm{ASA}$ & $1.03(0.90-1.16)$ & $0.89(0.77-1.01)$ & NS \\
\hline 4 & $\mathrm{ACh}+\operatorname{vitamin} \mathrm{C}$ & $1.58(0.88-2.28)$ & $0.79(0.66-0.91)$ & $\mathrm{p}<0.001$ \\
\hline \multirow[t]{3}{*}{5} & $\mathrm{NaCl}$ & $0.01(0.00-0.02)$ & $0.05(0.00-0.11)$ & NS \\
\hline & 3 (air) vs. 1 (air) & $p=0.006$ & & \\
\hline & $3\left(100 \% \mathrm{O}_{2}\right)$ vs. 1 (air) & & $\mathrm{p}<0.001$ & \\
\hline \multicolumn{5}{|c|}{$E D_{50}[m C]$} \\
\hline 1 & $\mathrm{ACh}$ & $2.25(1.84-2.75)$ & $2.21(1.79-2.74)$ & NS \\
\hline 2 & SNP & $4.21(3.71-4.77)$ & $4.21(3.84-4.62)$ & NS \\
\hline 3 & $\mathrm{ACh}+\mathrm{ASA}$ & $1.83(1.46-2.30)$ & $1.95(1.65-2.30)$ & NS \\
\hline 4 & $\mathrm{ACh}+\operatorname{vitamin} \mathrm{C}$ & $5.00(2.35-10.6)$ & $2.09(1.72-2.53)$ & $\mathrm{p}=0.002$ \\
\hline
\end{tabular}

Mean values (with 95\% CI in parentheses) are presented. p values are based on the comparison between air breathing and $100 \%$ oxygen breathing within each protocol. NS = Not significant; ASA = acetylsalicylic acid.

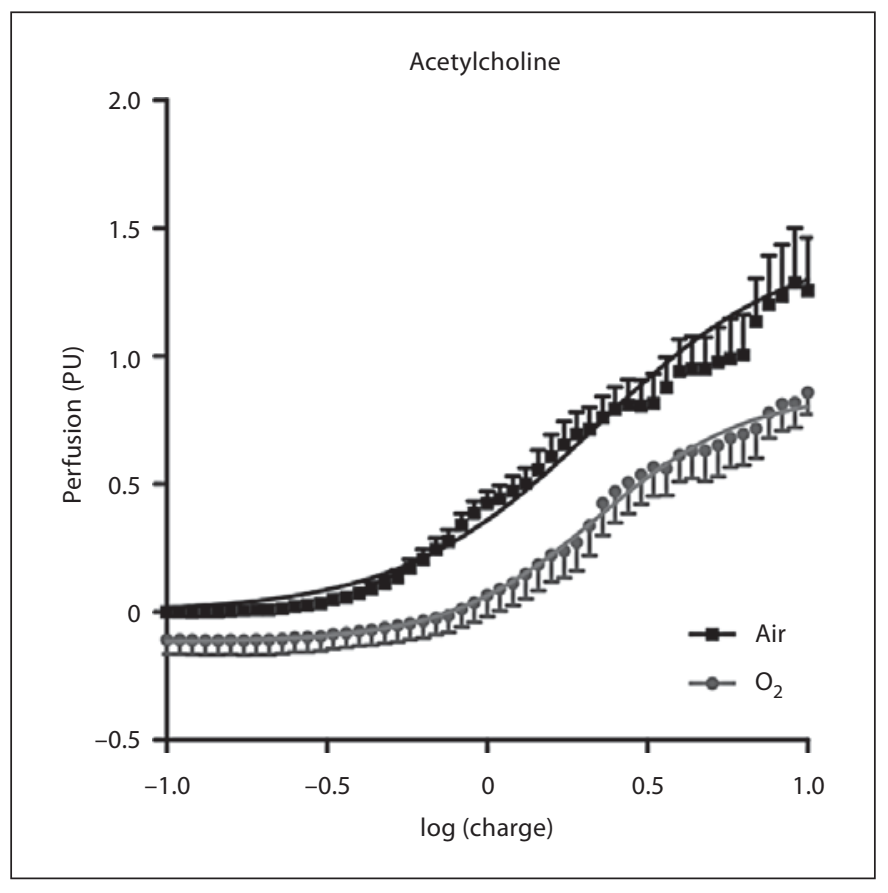

Fig. 1. Perfusion response to ACh delivered by anodal iontophoresis during 10 min of air breathing and 100\% oxygen breathing (protocol 1). A reduction of $30 \%$ in maximum perfusion increase was found during $100 \%$ oxygen breathing $(\mathrm{p}<0.001)$, while $\mathrm{ED}_{50}$ values were similar for the two responses.

\section{Baseline Perfusion}

In protocols 1 (ACh) and 2 (SNP), a decrease in mean baseline skin perfusion was found during breathing of oxygen $(\mathrm{p}<0.001)$. In the other protocols, mean baseline perfusion was similar during breathing of air and oxygen. There was no significant difference in baseline perfusion at the start of the air-breathing period between the protocols.

\section{Protocol 1: ACh}

In protocol $1(\mathrm{ACh})$, the maximum perfusion response was significantly lower while breathing oxygen than while breathing air [oxygen: $0.98(0.81-1.15)$ vs. air: 1.45 $(1.30-1.60) ; \mathrm{p}<0.001]$. The $\mathrm{ED}_{50}$ values did not differ significantly between results with breathing oxygen and air (fig. 1).

\section{Protocol 2: SNP}

During iontophoresis of SNP, the maximum responses were similar for breathing of oxygen and air [oxygen: $1.40(1.26-1.53)$ vs. air: 1.45 (1.28-1.62)]. Also, no difference was found between the $\mathrm{ED}_{50}$ values during breathing of oxygen and air [oxygen: $4.21(3.84-4.62)$ vs. air: 4.21 (3.71-4.77)] (fig. 2).

\section{Protocol 3: ACh + Aspirin}

When ACh iontophoresis was preceded by intake of aspirin, no difference in the maximum responses be- 


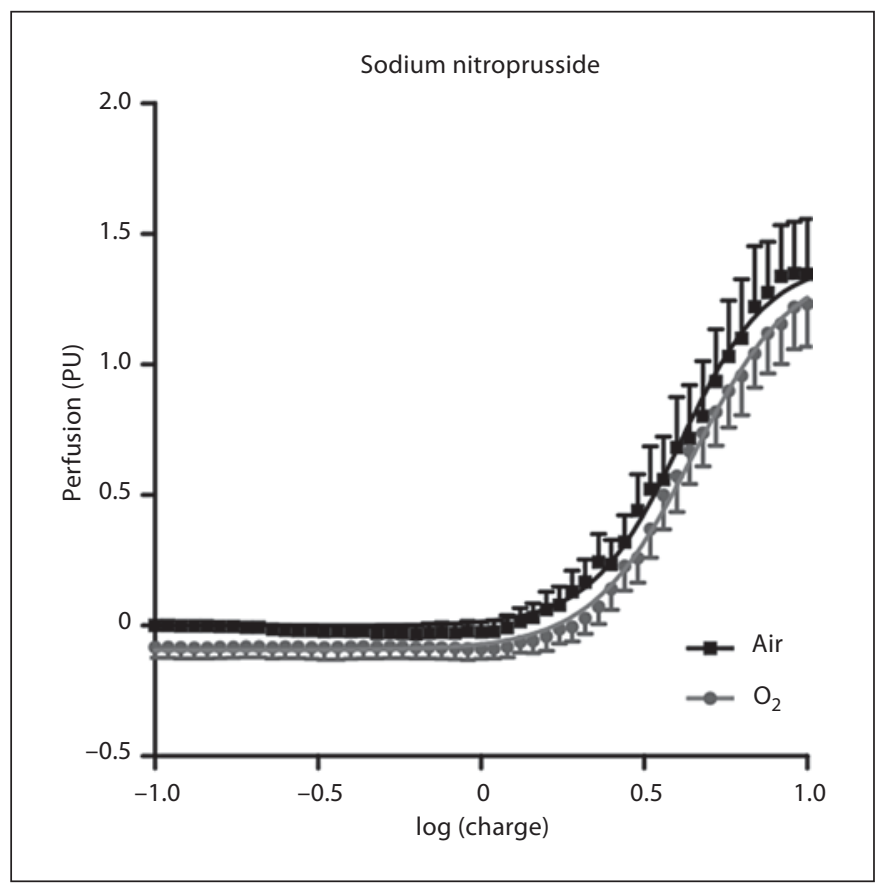

Fig. 2. Perfusion response to SNP delivered by cathodal iontophoresis during $10 \mathrm{~min}$ of air breathing and $100 \%$ oxygen breathing (protocol 2). No significant difference was found in dose-response parameters between air breathing and $100 \%$ oxygen breathing.

tween breathing oxygen and air was found [oxygen: 0.89 (0.77-1.01) and air: $1.03(0.90-1.16)]$. The $\mathrm{ED}_{50}$ values did not differ significantly between results with breathing oxygen and air (fig. 3). Maximum responses during air and oxygen breathing were significantly lower compared with untreated, air-breathing subjects $(\mathrm{p}=0.006$ and $\mathrm{p}<$ 0.001 , respectively).

\section{Protocol 4: ACh + Vitamin C}

When ACh iontophoresis was preceded by intake of vitamin $\mathrm{C}$, the perfusion response was reduced during oxygen breathing, but only at a charge of $6 \mathrm{mC}$ and above [oxygen: $0.79(0.66-0.91)$ vs. air: $1.58(0.88-2.28)]$. As a result of the absence of a plateau in the data obtained during breathing of air, the respective maximum response and $\mathrm{ED}_{50}$ value could not be estimated with high accuracy. However, the mean $\mathrm{ED}_{50}$ value was significantly lower during oxygen breathing than during air breathing [oxygen: 2.09 (1.72-2.53) vs. air: 5.00 (2.35-10.6)] (fig. 4).

\section{Protocol 5: Sodium Chloride}

When sodium chloride was delivered with iontophoresis to test for non-specific effects, no significant in-

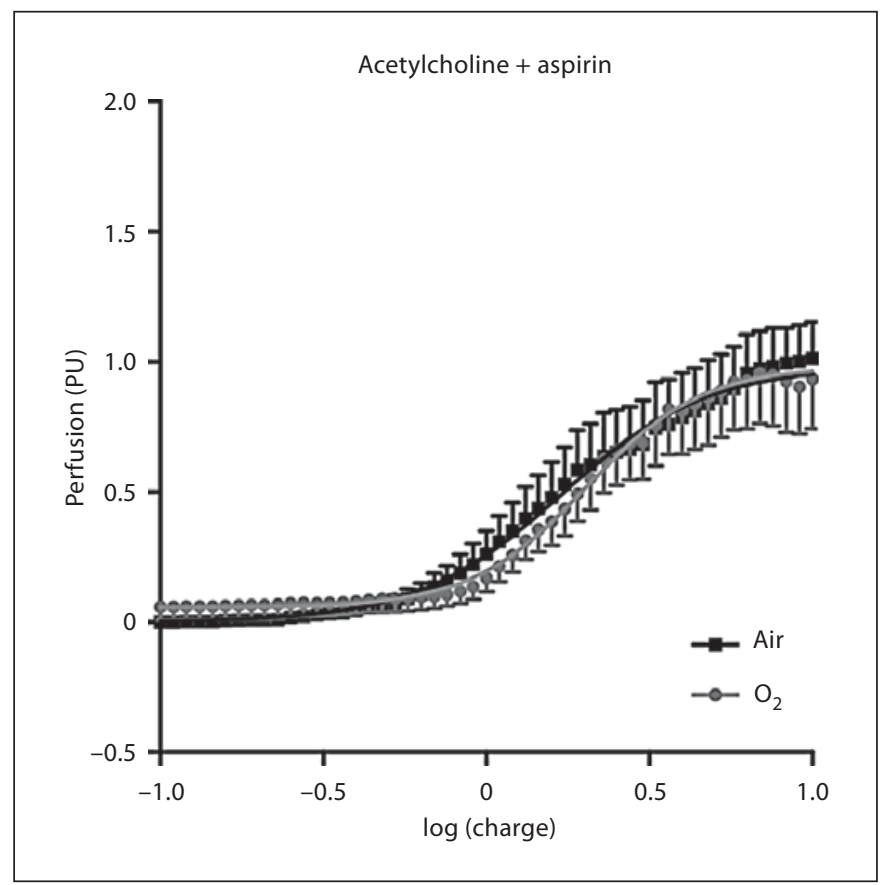

Fig. 3. Perfusion response to ACh delivered by anodal iontophoresis during $10 \mathrm{~min}$ of air breathing and $100 \%$ oxygen breathing. Synthesis of prostaglandins was inhibited by pre-treatment with aspirin (protocol 3). No significant difference was found in doseresponse parameters between air breathing and 100\% oxygen breathing. The maximum increase in perfusion was similar to the maximum perfusion increase to ACh during $100 \%$ oxygen breathing without pre-treatment (fig. 1).

crease in perfusion was found during breathing of air or oxygen (fig. 5).

In none of the protocols was there any difference in $\mathrm{ED}_{50}$ or maximum response between male and female subjects $(\mathrm{p}>0.32)$.

\section{Discussion}

The main findings in this study are that in healthy subjects, $100 \%$ oxygen breathing reduces the endothelium-dependent microvascular response to ACh with $30 \%$ compared to the response during normal air breathing. When subjects had been pre-treated with aspirin, there was no difference in response between oxygen breathing and air breathing. Instead, both responses were reduced with $30 \%$ compared to the response in untreated, airbreathing subjects. ACh-mediated vasodilatation during $100 \%$ oxygen breathing was partially restored after pretreatment with the antioxidant vitamin C. Breathing 


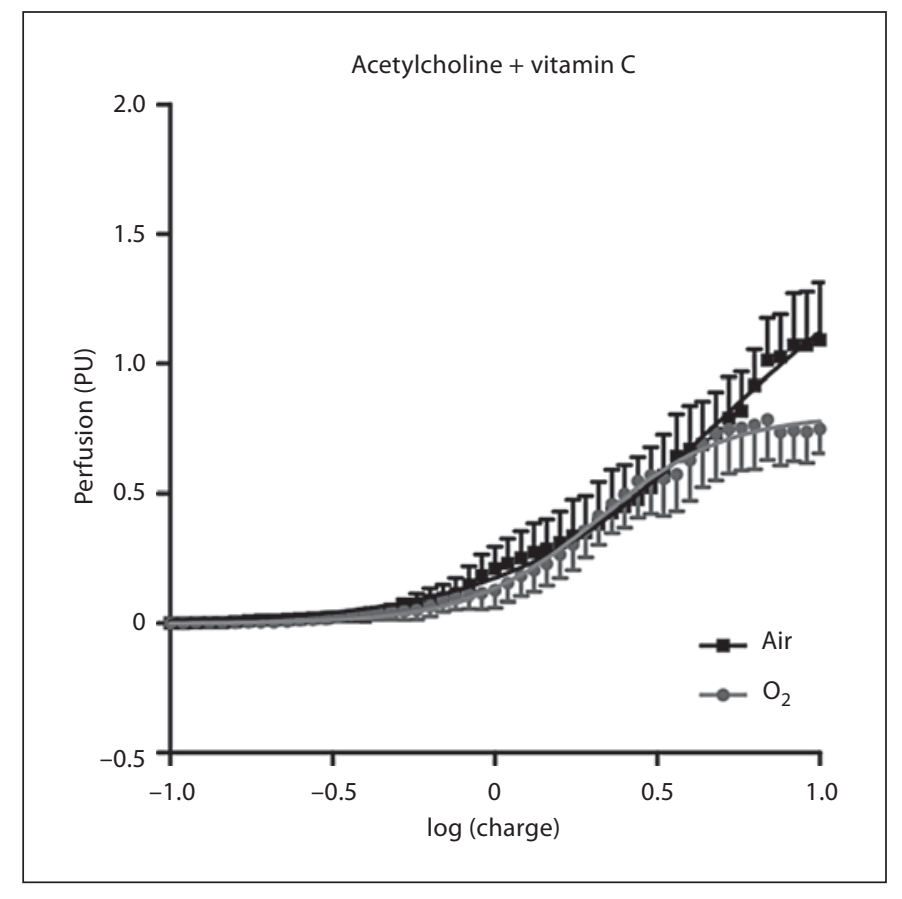

Fig. 4. Perfusion response to ACh delivered by anodal iontophoresis during $10 \mathrm{~min}$ of air breathing and $100 \%$ oxygen breathing after pre-treatment with vitamin C (protocol 4). No significant difference was found in dose-response parameters between air breathing and $100 \%$ oxygen breathing up to a charge of $6 \mathrm{mC}$ ( 5 min of oxygen breathing). At higher charges (longer time), the response to ACh during 100\% oxygen breathing did not increase further, while the response during air breathing continued to increase. Thus, the maximum increase in perfusion after pre-treatment with vitamin $\mathrm{C}$ was significantly higher for air breathing $(\mathrm{p}=0.0018)$.

$100 \%$ oxygen did not change the microvascular response to the NO donor SNP.

\section{Dose-Response Modelling}

The application of the $\mathrm{E}_{\max }$ model to perfusion response data obtained from a single-pulse iontophoresis protocol has been validated in previous studies by our group [22, 23]. This approach offers important advantages over more qualitative descriptions of responses. It enables a more powerful assessment of the dynamics of perfusion responses, as responses can be interpreted in terms of conventional pharmacodynamic parameters such as $\mathrm{ED}_{50}$ and maximum response. Also, modelling in tests of microvascular mechanisms may avoid confounding factors in the interpretation of responses [25].

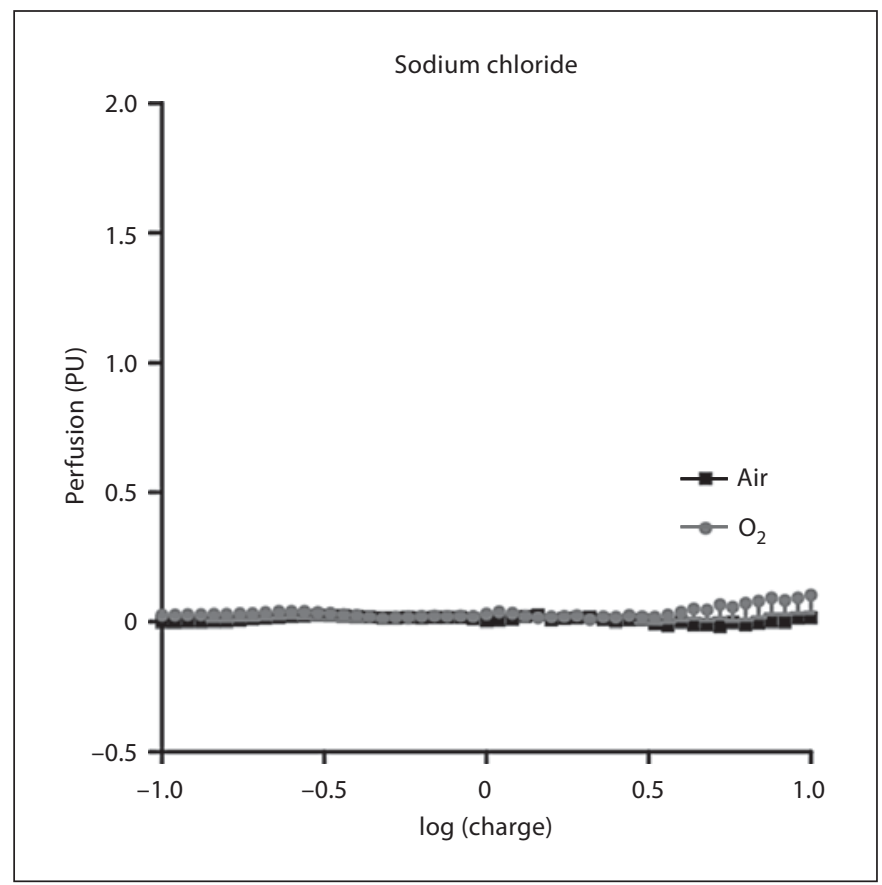

Fig. 5. Perfusion response to sodium chloride delivered by cathodal iontophoresis during $10 \mathrm{~min}$ of air breathing and $100 \%$ oxygen breathing. The perfusion did not change significantly, indicating that the protocols used in this study (single 10-min pulse, $0.02 \mathrm{~mA}$, total charge $12 \mathrm{mC}$ ) do not induce any non-specific responses.

\section{Effect of Oxygen on the Response to ACh}

When the subjects breathed $100 \%$ oxygen, the maximum response to ACh was reduced with $30 \%$ compared to the response during breathing of air, while the $\mathrm{ED}_{50}$ value was unchanged. This reduction was not found in the response to SNP. This finding confirms the results of a study by Yamazaki [13], in which breathing of 100\% oxygen reduced the vasodilatation in response to iontophoretically applied ACh, and suggests that the effect of hyperoxia is mediated by a non-competitive, endothelium-dependent mechanism.

\section{Effect of Aspirin on the Response to ACh}

The results of the present study indicate that prostaglandins take part in the ACh-mediated vasodilatation in the skin, since pre-treatment with aspirin resulted in a 30\% decrease in maximum response to the iontophoresis of ACh, when the subjects breathed air. Aspirin (acetylsalicylic acid) is a non-competitive, irreversible antagonist of the enzyme cyclooxygenase that inhibits the synthesis of prostaglandins by permanent acetyla- 
tion [26]. This finding is in line with a number of other studies, which have shown that the response to ACh in the cutaneous microvasculature is the result of at least two different mechanisms [13, 27-31]. The first mechanism is that ACh stimulates the activation of NO synthase (NOS, both neuronal and endothelial), by promoting the binding of a calmoduline- $\mathrm{Ca}^{2+}$ complex to NOS. The conversion of $\mathrm{L}$-arginine to form citrulline and $\mathrm{NO}$ is then catalysed by NOS. The second mechanism is the increased production of prostaglandins from the conversion of arachidonic acid by cyclooxygenase. Apart from the two mechanisms above, other mechanisms, such as endothelium-derived hyperpolarizing factor, may be responsible for the ACh-mediated vasodilatation.

Effect of Oxygen on the Response to ACh with Aspirin Firstly, when aspirin was given before the experiment and when subjects breathed air, a similar reduction (30\%) in maximum response was found compared to the ACh response during oxygen breathing, without pretreatment with aspirin. Secondly, under aspirin pretreatment, $100 \%$ oxygen breathing did not further reduce the response to ACh. Instead, both the maximum responses and the $\mathrm{ED}_{50}$ values for air and oxygen breathing were similar. These two results together strongly favour the hypothesis that the effect of hyperoxia on the endothelium-dependent vasodilatation involves prostaglandins.

Whether the interaction between hyperoxia and prostaglandins takes place during the synthesis or the action of prostaglandins is unclear. The role of prostaglandins in hyperoxia has been put forward by a number of previous studies. On the cell level, hyperoxia has been found to reduce cyclooxygenase activity [32]. In an in vitro model of isolated rat cremaster muscle arterioles, it was found that increasing oxygen tensions cause endothelium-dependent vasoconstriction, but that this was completely abolished in the presence of indomethacin, a cyclooxygenase inhibitor [12]. Furthermore, it has been shown that vasoconstricting prostanoids are released from the endothelium of the human umbilical vein at hyperoxia [14]. In healthy volunteers, hyperoxia significantly reduced the vasodilatation in response to isometric forearm contraction, but no further reduction was found after pre-treatment with aspirin [33]. These studies together with our present findings suggest that hyperoxia modulates the synthesis rather than the action of prostaglandins by inhibition of the cyclooxygenase pathway.

Microvascular Effects of Hyperoxia

\section{Effect of Oxygen on the Response to ACh with}

Vitamin $C$

In the present study, vitamin $C$ inhibited the effects of hyperoxia that were seen during iontophoresis of $\mathrm{ACh}$ up to an electrical charge of $5 \mathrm{mC}$ (or up to $250 \mathrm{~s}$ of iontophoresis). At higher charges, the maximum response was significantly lower during $100 \%$ oxygen breathing. Due to the absence of a clear plateau in the perfusion response during air breathing, it is difficult to compare the response to the corresponding responses in the untreated group, especially the $\mathrm{ED}_{50}$ values. However, the maximum responses were not significantly different from the maximum responses without vitamin $\mathrm{C}$ pretreatment, for both air breathing and 100\% oxygen breathing.

Vitamin C is a potent aqueous-phase antioxidant and it has been shown to lower oxidative stress in humans [34-36]. The molecular basis for its antioxidant effect is not fully understood, but there is some evidence that it increases the activity of superoxide dismutase (SOD) [37]. Extracellular SOD (SOD3) catalyzes dismutation of superoxide to hydrogen peroxide $\left(\mathrm{H}_{2} \mathrm{O}_{2}\right)$ and $\mathrm{O}_{2}$. By activating SOD3, vitamin $\mathrm{C}$ could reduce concentrations of superoxide and so augment the bioavailability of NO. Another plausible way for vitamin $\mathrm{C}$ to have an antioxidant effect is by lowering the activity of NADPH-oxidase, the enzyme responsible for the production of superoxide. This has been shown recently in animal models [38].

The finding that vitamin $\mathrm{C}$ partly abolishes the reduced endothelium-dependent vasodilatation during hyperoxia suggests that reactive oxygen species (ROS) are involved. Hyperoxia has been associated with increased production of ROS. Supporting evidence mainly comes from in vitro preparations $[15,39,40]$ and animal studies $[41,42]$. Recently, human studies have also been published in favour of a hyperoxic-ROS linkage $[43,44]$. In vitro studies have shown that hyperoxia results in the production of up to 2.5 times the normal concentrations of superoxide, which is probably the most important of the oxygen radicals [39].

From the present study it is impossible to determine by which mechanism ROS reduce the endothelium-dependent vasodilatation. The increase in superoxide may reduce the efficacy of the NO-producing chain, by either inhibiting activation of NOS or by lowering the availability of L-arginine through increased action of its degrading enzyme, L-arginase. This will lead to lower concentrations of NO and hence, less vasodilatation. However, the fact that during cyclooxygenase inhibition, $100 \%$ ox- 
ygen breathing did not further reduce the perfusion response compared with air breathing, suggests that there was no difference in the bioavailability of NO. On the other hand, based on the present results it could well be possible that ROS are mediators in the inhibiting effect of hyperoxia on prostaglandin production. In that case, vitamin $\mathrm{C}$ is indeed expected to reduce the inhibition of prostaglandin production during hyperoxia, through scavenging of these ROS.

In general, our observations are consistent with the result of a previous human study by Mak et al. [16]. In their study, ACh-mediated vasodilatation, measured by venous occlusion plethysmography in healthy subjects, was blunted by hyperoxia (inhalation of $100 \%$ oxygen for $10 \mathrm{~min}$ ). The hyperoxic vasoconstriction was then reversed by infusion of vitamin C. When an endotheliumindependent vasodilator was used (Verapamil), hyperoxia had no effect.

The results of a previous study by Waring et al. [4] are partially conflicting with our present findings. They investigated the effects of hyperoxia on perfusion after local application of ACh, SNP and a NOS inhibitor (LNMMA) in normal subjects using plethysmography. In their study there was a tendency towards a reduced response to ACh when breathing oxygen, but this was not significant. However, breathing oxygen did not alter perfusion in response to SNP or L-NMMA, suggesting that there was no significant involvement of NO [4], which is in agreement with our present results.

The reason why pre-treatment with vitamin $\mathrm{C}$ did not fully restore the reduced response during $100 \%$ oxygen breathing at higher charges remains unclear. It is possible that the local concentration of vitamin $\mathrm{C}$ in the skin was not sufficient to fully prevent the effect of ROS. The vitamin $\mathrm{C}$ dose, $2.5 \mathrm{~g}$ daily for 3 days, was chosen because higher doses do not increase the bioavailability of vitamin C, but only increase urinary excretion [21]. Also, single doses of $2 \mathrm{~g}$ have been shown to induce endothelial effects in man [45]. Therefore, it is unlikely that higher doses would have resulted in a fully restored perfusion response during $100 \%$ oxygen breathing. Neither is it likely that vitamin $\mathrm{C}$ had any direct systemic effects of its own, as short-term intake (hours to several days) is known to leave the cardiovascular system unaffected $[16,46]$. Apart from the possibility of incomplete inhibition of oxygen radicals, other effects may be responsible for the dependency of the effect of vitamin C on the iontophoretic charge, including more complex physiological interactions between vitamin C, ROS and prostaglandins.

\section{Study Limitations}

Limitations of this study include the fact that the effect of hyperoxia on microvascular responses was studied only indirectly, by observing the perfusion response in the forearm skin to $100 \%$ oxygen breathing during iontophoresis with endothelium-dependent and endothelium-independent drugs. A possible source of systematic error in our study is that oxygen was given in all cases after air had been breathed. However, this was done to avoid the need for washout periods.

Because the iontophoresis methodology itself has potent vasodilator effects, especially at the cathode, this could obviously be a confounding factor [19]. Our study protocol was chosen to minimize the effects of current, as has been earlier recommended by our group $[19,20]$. However, to investigate the importance of any non-specific effects, we used cathodal iontophoresis with sodium chloride, using the same settings as during iontophoresis of SNP. We found no significant increase in perfusion, which makes it unlikely that our results are affected by any non-specific effects of the iontophoresis current.

It should be stressed that the effects of hyperoxia found in this study may not be present at more modest hyperoxia levels, as the formation of superoxide may be more likely at higher oxygen concentrations. Further studies are required to elucidate the effects at modest hyperoxia levels. We did not measure concentrations of oxygen and carbon dioxide in blood. The model of oxygen delivery has, however, been used by our group with high reproducibility in which concentrations increased to a mean oxygen arterial partial pressure of $74 \mathrm{kPa}$ while breathing $100 \%$ in this non-rebreathing setup [47].

Studies have shown that the concentration of carbon dioxide decreases during hyperoxia, probably as a result of hyperventilation [48], which could have effects on vascular tone. The effects seem, however, to be modest, and possibly affect all oxygen measurements equally [3].

We did not consider the phase of the menstrual cycle of the female participants and the male/female balance was different for the different protocols. We have tested if $\mathrm{ED}_{50}$ and maximum response differed significantly between men and women in protocols 1 and 3 where the number of female subjects was largest. This was not the case. Also, we found that exclusion of all female subjects does not affect the conclusions drawn in this study. Nevertheless, an effect of gender has been suggested in different previous studies $[49,50]$ and we cannot entirely exclude the possibility that gender is of influence on the 
vascular mechanisms proposed in this study. Further investigations on a larger group of male and female subjects are needed to elucidate this issue.

\section{Conclusion}

This is the first completely non-invasive study that investigates the involvement of prostaglandins, $\mathrm{NO}$ and oxygen free radicals in the microvascular vasoreactivity during hyperoxia in healthy humans. We found that breathing $100 \%$ oxygen significantly attenuated that endothelium-dependent vasodilatation elicited by ionto- phoresis of ACh in the forearm skin of healthy subjects and that prostaglandins are involved in this attenuated response, as suggested by the lack of effect of hyperoxia on the response after pre-treatment with aspirin. Vitamin C partially abolished the attenuating effect of hyperoxia on ACh-mediated vasodilatation. Hyperoxia did not have any effect on endothelium-independent vasodilatation. These results indicate that hyperoxia inhibits the synthesis or action of vasodilator prostanoids by a mechanism that may involve ROS, and that hyperoxia is not likely to affect the production or the bioavailability of NO.

\section{References}

1 Daly WJ, Bondurant S: Effects of oxygen on the heart rate, blood pressure, and cardiac index of normal men-resting, with reactive hyperemia, and after atropine. J Clin Invest 1962;41:126-134.

$\checkmark 2$ Milone SD, Newton GE, Parker JD: Hemodynamic and biochemical effects of $100 \%$ oxygen breathing in humans. Can J Physiol Pharmacol 1999;77:124-130.

3 Rousseau A, Bak Z, Janerot-Sjoberg B, Sjoberg F: Acute hyperoxemia-induced effects on regional blood flow, oxygen consumption and central circulation in man. Acta Physiol Scand 2005; 183:231-240.

4 Waring WS, Thomson AJ, Adwani SH, Rosseel AJ, Potter JF, Webb DJ, Maxwell SR: Cardiovascular effects of acute oxygen administration in healthy adults. J Cardiovasc Pharmacol 2003;2:245-250.

5 Crawford P, Good PA, Gutierrez E, Feinberg JH, Boehmer JP, Silber DH, Sinoway LI: Effects of supplemental oxygen on forearm vasodilation in humans. J Appl Physiol 1997;2: 1601-1606.

6 Kenmure AC, Beatson JM, Cameron AJ, Horton PW: Effects of oxygen on myocardial blood flow and metabolism. Cardiovasc Res 1971;5:483-489.

7 Joachimsson PO, Sjoberg F, Forsman M, Johansson M, Ahn HC, Rutberg H: Adverse effects of hyperoxemia during cardiopulmonary bypass. J Thorac Cardiovasc Surg 1996; 112:812-819.

-8 Kiss B, Polska E, Dorner G, Polak K, Findl O, Mayrl GF, Eichler HG, Wolzt M, Schmetterer L: Retinal blood flow during hyperoxia in humans revisited: concerted results using different measurement techniques. Microvasc Res 2002;64:75-85.
9 Watson NA, Beards SC, Altaf N, Kassner A, Jackson A: The effect of hyperoxia on cerebral blood flow: a study in healthy volunteers using magnetic resonance phase-contrast angiography. Eur J Anaesthesiol 2000;17: 152-159.

10 Ellsworth ML, Forrester T, Ellis CG, Dietrich $\mathrm{HH}$ : The erythrocyte as a regulator of vascular tone. Am J Physiol 1995;269:H2155H2161.

11 Amin HM, Camporesi EM, Cohen D, Hakim TS, Kaniewski WS: Effects of acute exposure to hyperbaric oxygen on the rheology and morphology of the red blood cells in the rat. Microvasc Res 1995;50:417-428.

12 Messina EJ, Sun D, Koller A, Wolin MS, Kaley G: Increases in oxygen tension evoke arteriolar constriction by inhibiting endothelial prostaglandin synthesis. Microvasc Res 1994;48:151-160.

13 Yamazaki F: Hyperoxia attenuates endothelial-mediated vasodilation in the human skin. J Physiol Sci 2007;57:81-84.

14 Mildenberger E, Siegel G, Versmold HT: Prostanoids contribute to the oxygen-dependent regulation of vascular tone of human umbilical vein. J Perinat Med 2004;32: 149-154.

15 Rubanyi GM, Vanhoutte PM: Superoxide anions and hyperoxia inactivate endothelium-derived relaxing factor. J Appl Physiol 1986;250:H822-H827.

16 Mak S, Egri Z, Tanna G, Colman R, Newton GE: Vitamin C prevents hyperoxia-mediated vasoconstriction and impairment of endothelium-dependent vasodilation. Am J Physiol Heart Circ Physiol 2002;282:H2414H2421.

17 Wardell K, Jakobsson A, Nilsson GE: Laser Doppler perfusion imaging by dynamic light scattering. IEEE Trans Biomed Eng 1993;40: 309-316.
18 Morris SJ, Shore AC: Skin blood flow responses to the iontophoresis of acetylcholine and sodium nitroprusside in man: possible mechanisms. J Physiol 1996;496:531-542.

19 Droog EJ, Henricson J, Nilsson GE, Sjoberg F: A protocol for iontophoresis of acetylcholine and sodium nitroprusside that minimises nonspecific vasodilatory effects. Microvasc Res 2004;67:197-202.

-20 Droog EJ, Sjoberg F: Nonspecific vasodilatation during transdermal iontophoresis-the effect of voltage over the skin. Microvasc Res 2003;65:172-178.

21 Levine M, Conry-Cantilena C, Wang Y, Welch RW, Washko PW, Dhariwal KR, Park JB, Lazarev A, Graumlich JF, King J, Cantilena LR: Vitamin C pharmacokinetics in healthy volunteers: evidence for a recommended dietary allowance. Proc Natl Acad Sci USA 1996;93:3704-3709.

22 Henricson J, Tesselaar E, Persson K, Nilsson G, Sjöberg F: Assessment of microvascular function by study of the dose-response effects of iontophoretically applied drugs (acetylcholine and sodium nitroprusside) - methods and comparison with in vitro studies. Microvasc Res 2007;73:143-149.

-23 Tesselaar E, Henricson J, Jonsson S, Sjoberg F: A time-response model for analysis of drug transport and blood flow response during iontophoresis of acetylcholine and sodium nitroprusside. J Vasc Res 2008;46:270277.

24 Wagner JG: Kinetics of pharmacologic response. I. Proposed relationships between response and drug concentration in the intact animal and man. J Theor Biol 1968;20: 173-201.

25 Clough GF, Gush R: Is it important to model the impact of blood flow on the dose of drugs delivered transcutaneously? J Vasc Res 2009; 46:267-269. 
26 Harker L, Fuster U: Pharmacology of platelet inhibitors. J Am Coll Cardiol 1986;8:21B32B.

-27 Durand S, Tartas M, Bouyé P, Koïtka A, Saumet JL, Abraham P: Prostaglandins participate in the late phase of the vascular response to acetylcholine iontophoresis in humans. J Physiol 2004;561:811-819.

28 Holowatz LA, Thompson CS, Minson CT, Kenney WL: Mechanisms of acetylcholinemediated vasodilatation in young and aged human skin. J Physiol 2005;563:965-973.

29 Kellogg DL Jr, Zhao JL, Coey U, Green JV: Acetylcholine-induced vasodilation is mediated by nitric oxide and prostaglandins in human skin. J Appl Physiol 2005;98:629632.

30 Medow MS, Glover JL, Stewart JM: Nitric oxide and prostaglandin inhibition during acetylcholine-mediated cutaneous vasodilation in humans. Microcirculation 2008; 15 : 569-579.

-31 Lenasi H, Strucl M: The effect of nitric oxide synthase and cyclooxygenase inhibition on cutaneous microvascular reactivity. Eur J Appl Physiol 2008;103:719-726.

- 32 Ishii Y, Morita I, Murota S, Kitamura S: Hyperoxia decreases cyclooxygenase activity in endothelial cells. Prostaglandins Leukot Essent Fatty Acids 1993;48:455-461.

-33 Win TS, Marshall JM: Contribution of prostaglandins to the dilation that follows isometric forearm contraction in human subjects: effects of aspirin and hyperoxia. J Appl Physiol 2005;99:45-52.

- 34 Aghdassi E, Wendland BE, Steinhart AH, Wolman SL, Jeejeebhoy K, Allard JP: Antioxidant vitamin supplementation in Crohn's disease decreases oxidative stress: a randomized controlled trial. Am J Gastroenterol 2003;98:348-353.
35 Huang HY, Appel LJ, Croft KD, Miller ER 3rd, Mori TA, Puddey IB: Effects of vitamin $\mathrm{C}$ and vitamin $\mathrm{E}$ on in vivo lipid peroxidation: results of a randomized controlled trial. Am J Clin Nutr 2002;76:549-555.

36 Sanchez-Moreno C, Cano MP, de Ancos B, Plaza L, Olmedilla B, Granado F, Elez-Martinez P, Martin-Belloso O, Martin A: Pulsed electric fields-processed orange juice consumption increases plasma vitamin $\mathrm{C}$ and decreases F2-isoprostanes in healthy humans. J Nutr Biochem 2004;15:601-607.

37 Chen X, Touyz RM, Park JB, Schiffrin EL: Antioxidant effects of vitamins $\mathrm{C}$ and $\mathrm{E}$ are associated with altered activation of vascular NADPH oxidase and superoxide dismutase in stroke-prone SHR. Hypertension 2001;38: 606-611.

38 Ulker S, McKeown PP, Bayraktutan U: Vitamins reverse endothelial dysfunction through regulation of eNOS and $\mathrm{NAD}(\mathrm{P}) \mathrm{H}$ oxidase activities. Hypertension 2003;41: 534-539.

39 Parinandi NL, Kleinberg MA, Usatyuk PV, Cummings RJ, Pennathur A, Cardounel AJ, Zweier JL, Garcia JG, Natarajan V: Hyperoxia-induced $\mathrm{NAD}(\mathrm{P}) \mathrm{H}$ oxidase activation and regulation by MAP kinases in human lung endothelial cells. Am J Physiol Lung Cell Mol Physiol 2003;284:L26-L38.

40 Yusa T, Crapo JD, Freeman BA: Hyperoxia enhances lung and liver nuclear superoxide generation. Biochim Biophys Acta 1984;798: 167-174.

-41 Freeman BA, Crapo JD: Hyperoxia increases oxygen radical production in rat lungs and lung mitochondria. Biol Chem 1981;256: 10986-10992.

42 Jaensch S, Cullen L, Raidal SR: Normobaric hyperoxic stress in budgerigars: enzymic antioxidants and lipid peroxidation. Comp Biochem Physiol C Toxicol Pharmacol 2001; 128:173-180.
43 Carpagnano GE, Kharitonov SA, FoschinoBarbaro MP, Resta O, Gramiccioni E, Barnes PJ: Supplementary oxygen in healthy subjects and those with COPD increases oxidative stress and airway inflammation. Thorax 2004;59:1016-1019.

44 Vento M, Asensi M, Sastre J, Lloret A, Garcia-Sala F, Vina J: Oxidative stress in asphyxiated term infants resuscitated with $100 \%$ oxygen. J Pediatr 2003;142:240-246.

$\checkmark 45$ Levine GN, Frei B, Koulouris SN, Gerhard MD, Keaney JF, Vita JA: Ascorbic acid reverses endothelial vasomotor dysfunction in patients with coronary artery disease. Circulation 1996;93:1107-1113.

46 Eskurza I, Monahan KD, Robinson JA, Seals DR: Ascorbic acid does not affect large elastic artery compliance or central blood pressure in young and older men. Am J Physiol Heart Circ Physiol 2004;286:H1528-H1534.

47 Rousseau A, Abdiu A, Sjoberg F: Hyperoxaemia does not change concentrations of serotonin and $\beta$-thromboglobulin in blood of healthy humans. Scand J Clin Lab Invest 2004;64:81-85.

48 Dean JB, Mulkey DK, Henderson RA 3rd, Potter SJ, Putnam RW: Hyperoxia, reactive oxygen species, and hyperventilation: oxygen sensitivity of brain stem neurons. J Appl Physiol 2004;96:784-791.

49 Hendry RG, Marshall JM: Vasoconstrictor products of cyclo-oxygenase activity limit acetylcholine-induced cutaneous vasodilatation in young men. Clin Sci (Lond) 2004; 107:323-330.

50 Algotsson A, Nordberg A, Winblad B: Influence of age and gender on skin vessel reactivity to endothelium-dependent and endothelium-independent vasodilators tested with iontophoresis and a laser Doppler perfusion imager. J Gerontol A Biol Sci Med Sci 1995; 50:M121-M127. 